\title{
POBREZA Y EXCLUSIÓN SOCIAL COMO FORMAS DE VIOLENCIA ESTRUCTURAL \\ LA LUCHA CONTRA LA POBREZA Y LA EXCLUSIÓN SOCIAL ES LA LUCHA POR LA PAZ
}

\section{M. ${ }^{a}$ ASUNCIÓN MARTÍNEZ ROMÁN}

Departamento de Trabajo y Servicios Sociales. Universidad de Alicante.

\section{INTRODUCCIÓN}

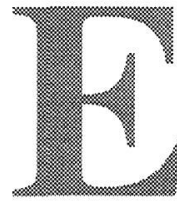

1 aumento de la pobreza, considerado globalmente, es un problema mundial de tal envergadura que ha pasado a ser un objetivo prioritario de Naciones Unidas. En el último Informe sobre Desarrollo Humano se constata que la pobreza «ya no es inevitable», tanto en el plano nacional como en el internacional, entendiendo la pobreza no sólo como pobreza de ingreso sino, en la perspectiva del desarrollo humano, «como denegación de opciones y oportunidades para vivir una vida tolerable» (vivir una vida larga, sana y creativa y disfrutar de un nivel decente de vida, libertad, dignidad, respeto por sí mismo y de los demás). El Informe destaca los avances logrados en todos los países del mundo en cuanto al ingreso y también en cuanto a la esperanza de vida y al acceso a los servicios sociales básicos, pero advierte que los adelantos han sido desiguales, con retrocesos, y que la pobreza sigue siendo generalizada (PNUD, 1997).

Si la pobreza es evitable, resulta ser más injusta la persistencia de situaciones como las destacadas en el Informe: más de la cuarta parte de la población del mundo en desarrollo sigue viviendo en la pobreza; alrededor de un tercio de la población (1.300 millones de personas) vive con un ingreso inferior a un dólar diario; la mayor cantidad de personas afectadas se encuentran en Asia meridional y Africa subsahariana; en América Latina y el Caribe la pobreza de ingreso afecta a unos 110 millones de personas y sigue creciendo; en Europa Oriental y los países de la Comunidad de Estados Independientes (CEI) se ha experimentado el mayor deterioro en los últimos diez años (120 millones de personas).

El problema también afecta a los países industrializados, en los que se calcula que más de 100 millones de personas viven por debajo 
de la pobreza de ingreso, siendo 37 millones las personas que carecen de empleo. Actualmente, en Estados Unidos, una cuarta parte de los niños viven en hogares que están por debajo de la línea de la pobreza. Este fenómeno de la «infantilización» de la pobreza se constata también en otros países industrializados. Así, alrededor de la mitad de los niños que viven en hogares con un sólo padre se hallan por debajo de la línea de pobreza en Australia, Canadá, Reino Unido y Estados Unidos, pero sólo el 5\% en Finlandia y el 8\% en Suecia (PNUD, 1997:87).

La pobreza y la exclusión social, en cuanto denegación de opciones y de oportunidades para vivir una vida tolerable, se analizan a continuación como una forma evidente de violencia estructural que puede y debe ser evitada.

\section{HAY VIOLENCIA CUANDO LAS PERSONAS ENCUENTRAN OBSTÁCULOS PARA SU DESARROLLO PERSONAL Y SOCIAL.}

Galtung, experto en temas de investigación para la paz, partiendo de una definición generalista de la paz como ausencia de violencia, sostiene una concepción amplia de la violencia, más allá de la mera violencia física, en la que se relacionan directamente paz y desarrollo. La paz, en sentido amplio, es la ausencia tanto de violencia directa como de violencia estructural entre los Estados y dentro de los Estados, lo que supone la posibilidad de lograr la satisfacción de las necesidades básicas: la supervivencia, el bienestar, la identidad y la libertad para todos. El desarrollo, en sentido restringido, es igual a crecimiento económico y, en sentido amplio, es igual a la satisfacción de las necesidades humanas básicas: supervivencia, bienestar, identidad y libertad para todos (Galtung, 1985:107).

Es decir, la ausencia de paz, la violencia, está directamente relacionada con obstáculos o impedimentos a las posibilidades de desarrollo humano. En consecuencia, este autor considera que «la violencia está presente cuando los seres humanos se ven influidos de tal manera que sus realizaciones efectivas, somáticas y mentales, están por debajo de sus realizaciones potenciales», de modo que «cuando lo potencial es mayor que lo efectivo, y ello es evitable, existe violencia» (Galtung, 1995:314-315).

La pobreza (miseria), según Galtung, es una forma de sufrimiento, es la privación de las necesidades básicas implantada en la estructura y justificada por la cultura. Según la tipología del autor, la pobreza puede considerarse una forma de violencia estructural o indirecta, situación en la que la violencia se genera y está implantada dentro de la 
estructura y se manifiesta como un poder desigual y, consiguientemente, como oportunidades de vida distintas. La pobreza se apoya en la violencia cultural, es decir, la religión, la ideología, la lengua, el arte, la ciencia o la cosmología pueden servir para justificar y legitimar la existencia de la pobreza, considerándola algo inevitable (Galtung, 1996) y hasta, incluso, necesario (Tortosa, 1993:53).

En consecuencia, la pobreza es una forma de violencia institucionalizada e interiorizada, de ahí las dificultades para luchar contra ella, ya que cualquier intento de erradicarla producirá fuertes resistencias, tanto por parte de las estructuras sociales que se resistirán a los cambios, como por parte de los propios sujetos afectados. Las personas que carecen de poder de decisión sobre la distribución de los recursos existentes no tienen las mismas oportunidades de acceso a los medios precisos para desarrollar sus potencialidades personales. Esta diferencia de oportunidades origina una permanente situación de desigualdad que puede calificarse como violencia estructural o injusticia social, violencia que, a su vez, puede ser manifiesta o latente (Galtung, 1995:320).

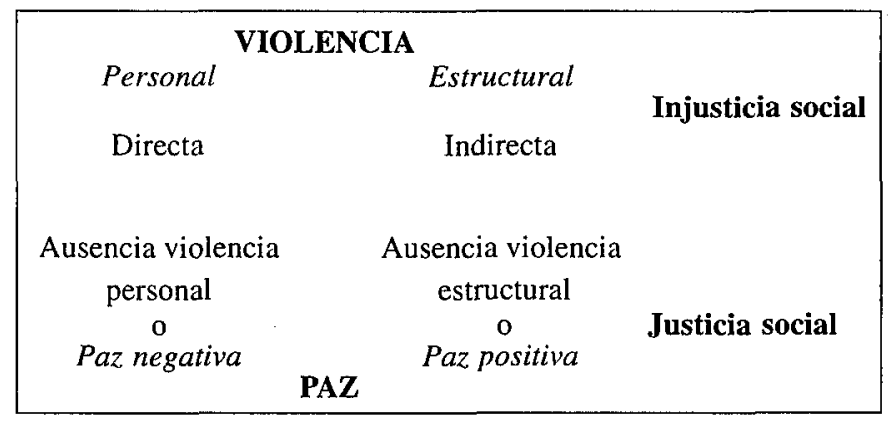

FUENTE: Galtung, J. (1995) Investigaciones teóricas. Sociedad y Cultura contemporáneas. Madrid, Tecnos-Instituto de Cultura «Juan Gil-Albert», pág. 348.

Así, la ausencia de violencia estructural o paz estructural (paz positiva) sería la existencia de justicia social, es decir, una distribución igualitaria del poder y de los recursos, lo que lleva al autor a destacar la estrecha relación entre este tipo de paz y la teoría del desarrollo, tal como la más reconocida relación entre la ausencia de violencia personal (paz negativa) y la teoría del conflicto. Por lo tanto, en la investigación para la paz tiene un lugar necesario la investigación del desarrollo.

Desde diferentes organismos internacionales, se destaca, en los últimos años, tanto la existencia en el mundo de la pobreza, la injusticia social y el deterioro ambiental como la ausencia de su justificación, 
estableciendo la relación causal entre pobreza y crecimiento económico e, incluso, con los conflictos bélicos. Situación que, si bien afecta en mayor medida a países en proceso de desarrollo, afecta también a los países industrializados.

En la Conferencia sobre Población y Desarrollo de El Cairo se reconocía que «la pobreza generalizada sigue siendo el principal problema con que se tropieza al efectuar actividades de desarrollo. La pobreza suele ir acompañada de desempleo, malnutrición, analfabetismo, el bajo nivel social de la mujer, riesgos ambientales y un acceso limitado a servicios sociales y sanitarios, incluidos los servicios de salud reproductiva, que a su vez, incluye la planificación de la familia. Todos estos factores contribuyen a elevar los niveles de fecundidad, morbilidad y mortalidad, así como a reducir la productividad económica. La pobreza también está íntimamente relacionada con la inadecuada distribución espacial de la población, el uso insostenible y la distribución no equitativa de recursos naturales tales como la tierra y el agua y una grave degradación del medio ambiente» (Naciones Unidas, 1994:18).

Asimismo, la Comisión Sudamericana de Paz destaca que «la pobreza, la exclusión social y el desempleo son factores de inestabilidad económica, política, social y cultural que generan violencia y amenazan la seguridad de las personas» (Comisión Sudamericana de $\mathrm{Paz}$, Seguridad y Democracia, 1994). El Informe sobre Desarrollo Humano de 1994 destaca el interés de la ONU por un desarrollo humano sostenible, resaltando que el programa de paz y el programa de desarrollo deben ser integrados en última instancia: «sin paz no puede haber desarrollo; pero sin desarrollo la paz puede estar amenazada» (PNUD, 1995). Una condición imprescindible para que se dé la paz es el hecho de que los seres humanos tengan seguridad en sus vidas cotidianas, más allá de la clásica concepción de la seguridad como protección del territorio nacional ante un posible conflicto bélico contra otro país. Esta nueva concepción de la seguridad humana no es un concepto meramente defensivo sino integrador, que reconoce la preocupación de las personas ante sus condiciones de vida diarias, lo que les lleva a entender la seguridad como la protección ante acontecimientos que pueden tener gran trascendencia en sus vidas, tales como la enfermedad, el hambre, el desempleo, etc.

Por lo tanto, al pensar en la seguridad no hay que pensar sólo en la seguridad militar sino también en la seguridad humana o, en lo que es lo mismo, preocuparse por la seguridad de la población entendiendo por tal las condiciones de vida y la dignidad humanas. En el primer caso, se busca protección ante posibles riesgos que pondrían en peligro a seres humanos, en el segundo, hay que preocuparse de las con- 
diciones de vida que impiden a las personas su desarrollo humano, se centra en el ser humano. La seguridad es un concepto integrador y globalizador en el que se pueden distinguir siete categorías: seguridad económica, seguridad alimentaria, seguridad en materia de salud, seguridad ambiental, seguridad personal, seguridad de la comunidad y seguridad política.

De todo lo dicho se desprende la interdependencia existente entre seguridad humana y desarrollo humano, si bien hay que distinguir las diferencias: el desarrollo humano es un concepto más amplio, como un proceso de ampliación de la gama de opciones de las que dispone la gente; la seguridad humana «hace posible que la gente pueda ejercer esas opciones en forma segura y libre y que pueda tener relativa confianza en que las oportunidades que tiene hoy no desaparecerán totalmente mañana» (PNUD, 1995:26-27). Las limitaciones o déficits del desarrollo humano tienen como consecuencia la pérdida o inexistencia de la seguridad humana. A su vez, la existencia de inseguridad humana puede ser una causa de generación de violencia por parte de las personas que se ven privadas de su seguridad y ven mermadas o anuladas sus oportunidades de desarrollo personal, de lo que se pueden encontrar ejemplos en todos los países (Rifkin, 1996:249-258).

Esta falta de seguridad humana no sólo afecta a los países en proceso de desarrollo sino que, dadas las características del sistema mundial, cuando la inseguridad puede traspasar las fronteras nacionales también puede verse amenazada la seguridad humana a escala mundial. Las amenazas contra la seguridad humana mundial del siglo XXI, como crecimiento descontrolado de la población; disparidad de oportunidades económicas; migración internacional excesiva; deterioro del medio ambiente; producción y tráfico de estupefacientes; y terrorismo internacional, plantean la necesidad de actuar conjuntamente todos los países, al no ser posible para ninguno de ellos permanecer aislado del resto.

Resulta nuevo el mensaje de Naciones Unidas sobre lo injustificado de la existencia de situaciones de pobreza humana cuando, durante años, se ha venido justificando la existencia de situaciones de pobreza como el costo inevitable de todo proceso de desarrollo económico argumentándose que, cuando se lograse dicho desarrollo, los beneficios alcanzarían a los más pobres que se beneficiarían también de ello. Sin embargo, el tiempo ha demostrado que dicho planteamiento sólo asegura el beneficio de unos pocos en los que se acaban concentrando las ganancias, mientras que, por el contrario, un número mucho mayor de personas no llegan a alcanzar las mejoras logradas e, incluso, pueden ver empeorar sus condiciones de vida como precio de la mejora del primer grupo. 
La situación resulta más injusta, socialmente hablando, cuando las ganancias económicas se concentran cada vez en menos manos, en proporciones escandalosas que suponen una seria amenaza social. Así, en 1994, la relación entre el ingreso del $20 \%$ más rico del mundo y el ingreso del $20 \%$ más pobre era de 78 a 1, en comparación con 61 a 1 en 1991 y con 30 a 1 en 1960 (PNUD, 1997:29).

\section{III.POBREZA Y EXCLUSIÓN SOCIAL COMO FORMAS DE VIOLENCIA ESTRUCTURAL.}

Desde 1990, el Programa de Naciones Unidas para el Desarrollo viene proponiendo un nuevo paradigma de desarrollo por el que se sitúa al ser humano en el centro del proceso de desarrollo, destacando que el crecimiento económico no es un fin sino un medio necesario para el desarrollo humano. El Informe sobre el Desarrollo de 1994, señala que un desarrollo humano significa la posibilidad de que toda persona amplíe plenamente su capacidad humana y la aproveche al máximo en todas las esferas (económica, social, cultural y política), protegiendo las opciones de las generaciones futuras (desarrollo sostenido) y asignando prioridad a la reducción de la pobreza, promoción de empleo productivo, promoción de la integración social y regeneración del medio ambiente (PNUD, 1995:5).

Se define el Desarrollo Humano como el proceso de ampliación de opciones de la gente, siendo las opciones fundamentales: vivir una vida larga, sana y creativa; tener educación (conocimientos y capacidad de comunicación); y disfrutar de un nivel de vida decente. Además, son necesarias otras opciones como: libertad política; garantía de otros derechos humanos; respeto por sí mismo, así como recibir el respeto de los demás (PNUD, 1997:17-18).

Cuando hay privación de estas opciones hay pobreza humana y ello es socialmente injusto, dado que otras personas sí disponen de esas opciones y, por lo tanto, de un desarrollo humano. Al hablar de pobreza humana se alude a privación de capacidades básicas, es decir, la privación en años de vida, salud, vivienda, conocimientos, participación social, seguridad personal y medio ambiente. Desde la perspectiva de la capacidad, la pobreza de una vida se basa no sólo en la situación empobrecida en la que una persona vive efectivamente, sino también en la carencia de oportunidad real, determinada por limitaciones sociales y por circunstancias personales para vivir una vida «valiosa y valorada». Esta otra cara de la pobreza es la que nos muestra las dificultades de integración social que encuentran muchas personas, lo que nos sitúa en la esfera de la exclusión social. 
Siguiendo a Rodgers, podemos considerar la exclusión social como una característica de los individuos o como una característica de las sociedades. En el primer caso, la exclusión social se centra en las condiciones de vida que la gente tiene. Una persona socialmente excluida está socialmente aislada en algún sentido; puede tener desventaja en cuanto al reconocimiento de sus derechos legales o respecto a su capacidad para ejercerlos efectivamente, entrando en una dinámica de causalidad acumulativa, de efectos negativos que refuerzan la desventaja y que pueden hacerla irreversible. En el segundo caso, se puede considerar la exclusión social con un enfoque institucional (instituciones, normas etc.), el marco en el que los individuos se relacionan. En este sentido, hay exclusión social cuando la sociedad permite diferentes formas de discriminación, cuando se niega a individuos y a grupos el acceso a los bienes, servicios, mercados y recursos que se asocian a la ciudadanía. En resumen, puede verse la exclusión social como un concepto multidimensional de pobreza que introduce aspectos de participación social y de realización de derechos como seguridad (física, de sustento y de protección ante contingencias, protección, identidad y plena ciudadanía) en su conceptualización (Rodgers, 1995:43-55).

La exclusión social es también una forma de violencia estructural en cuanto entre las causas de la exclusión encontramos fenómenos estructurales, tal como se reconoce desde las Comunidades Europeas: la persistencia del desempleo de larga duración; las consecuencias para el mercado laboral de las mutaciones industriales (especialmente para los trabajadores menos cualificados); el deterioro de las estructuras familiares; la evolución del sistema de valores, en particular, el hundimiento de la cohesión y de las formas tradicionales de solidaridad; la tendencia a la fragmentación social y, como consecuencia, la reducida participación en las instituciones representativas tradicionales; y la evolución de los fenómenos migratorios. El Comité Económico y Social considera que el problema de la exclusión social en Europa es un problema de grandes dimensiones, que supone una amenaza al progreso social y económico europeo, a la unidad europea y nacional e, incluso, para la propia democracia (Comunidades Europeas, 1993).

De la serie de fenómenos citados como factores estructurales generadores de la exclusión social, hay que destacar por su importancia el desempleo de larga duración y la inestabilidad/precariedad laboral, que han dado lugar a la aparición de nuevos grupos de población en situación de precariedad y de pobreza, afectando especialmente a jóvenes, mujeres, minorías étnicas y mayores de 45 años. Un reciente informe de la OIT estima que, en 1996, un 30\% de la población acti- 
va mundial estaba en desempleo o sub-empleada y que el número de los trabajadores pobres (insuficientemente remunerados) aumentaba sin cesar (OIT, 1997).

La Unión Europea no está libre de fenómenos como la mundialización de la economía y la globalización de la tecnología que han originado, por una parte, la demanda de una mayor cualificación laboral y, por otra, la desvalorización de los trabajadores no cualificados con la consiguiente desaparición de gran número de empleos. En consecuencia, en los países industrializados ha tenido lugar una situación generalizada de desempleo estructural que ha perdurado durante los 80 , con un alto costo social para muchas personas que han pasado a ser pobres o tienen gran peligro de serlo por su vulnerabilidad.

La novedad es que estas personas que han pasado a estar en peligro de llegar a ser pobres pertenecen a las clases medias formadas por trabajadores que se habían beneficiado de los modos de crecimiento económico anteriores cualificándose y empleándose, con lo que contribuían a mantener los sistemas de protección social diseñados precisamente para evitar que se llegaran a encontrar en una situación de pobreza.

Engelhard señala tres consecuencias de esta situación mundial:

a) las personas son valoradas socialmente en función de su capacidad de ser productivas y de su poder de consumo y ahorro, por lo que las personas que no pueden acceder a un empleo pasan a estar en riesgo o en situación de exclusión social;

b) la competitividad y la eficacia tecno-económica son los criterios financieros dominantes, buscándose la máxima ganancia a pesar de los costes sociales;

c) el debilitamiento de lo político, como resultado de la primacía de lo económico sobre lo social y de lo financiero sobre lo económico (Engelhard, 1996:36-41).

El desempleo es, además, un factor generador de una nueva categoría de personas pobres denominados por Castel «los válidos invalidados por la coyuntura» que, si no trabajan, no es porque no quieran hacerlo sino porque no encuentran trabajo y a los que tampoco se les puede «rehabilitar» en cuanto que no son encuadrables en las categorías clásicas de la ayuda social. Hay que ayudarles a encontrar un lugar «normal» en la sociedad y ello sólo será posible a través de un empleo (Castel, 1995).

Castel destaca la necesidad de analizar el trabajo como el medio actual de situarse en la estructura social, ya que existe una fuerte correlación entre el lugar ocupado en la división social del trabajo y la participación en las redes sociales y en los sistemas de protección que cubren a un individuo ante las contingencias a lo largo de su existen- 
cia. El aumento de la vulnerabilidad de la población general supone un aumento de la fragilidad de las personas que ya eran antes vulnerables, encontrándose una correlación entre la degradación del status concedido por el trabajo y la fragilización de los soportes relacionales familiares, vecinales y sociales (Castel, 1995:415). Para representar esta situación, el autor recurre a establecer unas «zonas» de cohesión social en las que se situarían las personas en función de su situación laboral y social, con la posibilidad de sub-zonas y variaciones en función de las situaciones personales y sociales.

Son tres las zonas en que se pueden encuadrar a las personas en relación a la cohesión social: una primera zona, denominada de «integración» en la que se encuentran las personas que disfrutan plenamente de los derechos de ciudadanía; una segunda zona en la que se encuentran las personas que se encuentran en situación de «vulnerabilidad», con grave riesgo de empobrecimiento y exclusión social y, finalmente, la zona en la que se encuentran las personas en situación de «exclusión social» (Castel, 1995:474).

Partiendo de esta propuesta de Castel, podemos sintetizar la situación de las personas en función de las características diferenciales que implican la adscripción a cada una de las zonas:

1. Zona de INTEGRACIÓN.
empleo estable,
relaciones familiares y sociales fuertes.
Ingresos económicos, status social, protección social, apoyo redes sociales, opor-
tunidades de participación en la vida social, cultural, económica y política, junto
con habilidades para ejercerla efectivamente.
2. Zona de VULNERABILIDAD.
empleo precario,
soportes familiares y sociales frágiles.
Inestabilidad laboral, paro recurrente, ingresos insuficientes, débil protección so-
cial, «vivir al día» o solamente sobrevivir, peligro de endeudamiento, debilita-
miento de las redes sociales primarias, inicio del sentimiento de inutilidad social y
de un proceso de desvalorización personal y social.
3. Zona de $E X C L U S I O ́ N$.


En resumen, el trabajo es el principal fundamento de la ciudadanía, en tanto comporta una dimensión económica y una dimensión social, es el medio concreto sobre el que se construyen los derechos y los deberes sociales. Hay que destacar la denominada zona de «vulnerabilidad», caracterizada por la situación de precariedad laboral y social, en la que se podría encuadrar a un importante grupo de población. El hecho de que sea situable en esta zona una parte importante de la población, debería convertirla en diana para la intervención de las políticas sociales si se deseara evitar la exclusión social.

En el caso español, se observa un fenómeno similar al descrito por Castel: el desempleo y el subempleo son uno de los principales problemas sociales actuales, existiendo un gran grupo de personas con graves dificultades para subsistir y, otros muchos, en precaria situación (Martínez Román, 1996a). Entre los distintos factores explicativos de la agravación del desempleo español en los últimos años hay que citar: por una parte, la disminución del consumo privado debido a la fuerte moderación salarial, el aumento del paro y los recortes en las prestaciones por desempleo, con efectos agravados por los recortes del gasto público; por otra, una serie de efectos estructurales como la destrucción de millones de puestos de trabajo consecuencia de la reestructuración industrial y factores estructurales de la economía española que la sitúan en débil posición en el contexto mundial. En opinión de Recio, las políticas laborales adoptadas al primar el recorte de salarios y la progresiva reducción de los derechos laborales, pueden haber acentuado las deficiencias del mercado laboral español y de su sistema productivo, potenciando crecientes desigualdades sociales que han generado un clima de tensión social (Recio, 1997:276-277).

Como se ha visto anteriormente, la exclusión social no es consecuencia, únicamente, de la falta de medios económicos; en el fenómeno inciden factores de muy diversa índole, entre los cuales juega un papel fundamental la dificultad para incorporarse al mercado de trabajo, la posibilidad de lograr el pleno desarrollo como persona y la satisfacción de las necesidades de forma autónoma y por medios lícitos. El hecho de llevar tiempo fuera del sistema de producción implica una cierta desadaptación al sistema de vida normalizado, con dificultad para acceder a los servicios, a la participación en la vida social y con un lógico desfase en la preparación necesaria para encontrar nuevamente trabajo; además de la pérdida de conocimientos y habilidades para el trabajo, se produce una frustración y un deterioro progresivo de la actitud frente al trabajo.

Los aspectos personales del paro «tienen repercusiones físicas (consumo de alcohol, incremento de suicidios y para-suicidios, alteraciones psicopatológicas, etc), también deja huella en las relaciones 
familiares y en las sociales, tales como la disminución del uso de habilidades sociales que provoca, la disminución de posibilidades de contacto interpersonal o la carencia de la fuente de estima pública que reporta el ejercicio laboral» (Quevedo, 1990: 211). La prolongación de esta situación provoca un sentimiento de pérdida de confianza en uno mismo y en la sociedad, que puede traer consigo problemas adicionales de salud y sociales que pueden empujar, cada vez más, a mantenerse al margen de la sociedad. A falta de estudios conocidos en nuestro país, hay que recordar, por ejemplo, la correlación entre crecimiento de desempleo y violencia criminal que queda demostrada en el estudio de Merva y Fowles, en Estados Unidos, encontrando que un crecimiento de un $1 \%$ en el desempleo supone un crecimiento del $6,7 \%$ en los homicidios, de un 3,4\% en los crímenes violentos y de un $2,4 \%$ en los crímenes contra la propiedad (Merva y Fowles, 1992).

En opinión de Cáritas Española, dos causas importantes del aumento del grupo de personas excluidas han sido «la flexibilización del mercado de trabajo, con la apertura constante de «huecos», que no ha conseguido que el grupo de los parados de larga duración encuentre un lugar en él y el recorte de los subsidios que provoca que un grupo de trabajadores en paro caigan en situaciones de exclusión, sin posibilidades de incorporarse al mundo laboral» (Pérez, 1996:15). Opinión que coincide con otras realizadas por otras organizaciones, entre ellas los sindicatos. Un caso especial es la aparición de los nuevos demandantes de ayuda económica, sin historia previa de necesidad económica, que como consecuencia de la crisis económica «de la noche a la mañana» pierden sus fuentes de ingresos y, con ellos, un nivel de bienestar, pasando a no saber cómo subsistir. Cáritas cifra esta cara de la pobreza entre un $10 \%$ y un $14 \%$ de las demandas, registradas a través de sus servicios, que corresponden a personas de clase media, y que llegan muchas veces derivados desde los Servicios Sociales públicos, impotentes para ayudarles. Para Cáritas es importante destacar la peculiaridad de estas situaciones, que quedarían superadas con la posibilidad de un empleo (no hay historia previa de marginación), por lo que, en su opinión, estas situaciones tienen su origen en los desequilibrios estructurales inherentes al modelo económico actual (generador de paro y de inestabilidad social) y en las carencias de una política social incapaz de prevenir la aparición de nuevos grupos de desprotegidos (Cáritas Española, 1997).

Resumiendo, el desempleo y el subempleo han llegado a ser en estos momentos la mayor preocupación de los españoles, en unos casos, por estar sufriendo directamente sus consecuencias y, en otros, por la pérdida generalizada de la seguridad asociada, tradicionalmente, a muchos empleos o por la dificultad de acceder a un primer em- 
pleo. En este contexto difícil para el conjunto de la población, hay que destacar las especiales dificultades de las personas incluidas en la categoría de los «vulnerables» (Tortosa, 1993:61-64), aquellas categorías sociales producto de la desigualdad, con menos poder, que suponen, en este contexto de dificultad general de empleo, un aumento de la vulnerabilidad de determinados grupos de población como son la edad, el género, la existencia de discapacidades, inmigración, minoría cultural, etc. Por razones de espacio tan sólo podemos comentar, brevemente, la situación en razón de edad y género.

En relación a la edad, un caso especial es el de los jóvenes españoles cuyo mayor problema es el desempleo o el subempleo, estimándose en 1.120.000 los jóvenes sin empleo, las cifras más altas de la Unión Europea (Centro de Estudios del Cambio Social, 1997: XXIII). En 1995, se constató una disminución del número de jóvenes protegidos ante el desempleo (16-34 años). Del total de 177.316 perceptores menos en media anual perceptores de subsidio, 144.588 fueron jóvenes, entre los perceptores de prestaciones contributivas el descenso del número de perceptores no presenta variaciones por grupos de edad (CES, 1996:309-310). En 1996, los parados menores de 30 años son la mitad del total de los que, a su vez, la mitad llevaba más de un año buscando empleo; de los que han logrado trabajo, un tercio ha conocido cuatro trabajos o más (CES, 1997:86-190).

Estos problemas deben analizarse en el contexto de la situación general del empleo y, por tanto, no se trata de exigir simplemente a los jóvenes un cambio por su parte. La mayoría de las ofertas a las que los jóvenes ha podido optar en los últimos años han sido temporales, de corta duración y con escasas o nulas posibilidades de protección social, ocupando muchas veces empleos de inferior cualificación con lo que, además, se relega a otros jóvenes sin cualificación que ven disminuidas sus oportunidades. En opinión de los sindicatos mayoritarios uno de los factores que explica esta situación de los jóvenes es la implantación, en la reforma laboral de 1993, de la figura del contrato de aprendizaje, que ha degradado las condiciones laborales de los jóvenes sin garantizar realmente la formación, y la deslaboralización de los contratos de menos de $48 \mathrm{~h} . /$ mes o de menos de $12 \mathrm{~h} . / \mathrm{semana}$ (Comisión de Trabajo y Seguridad Social del Senado, 1994:10-19; Elvira, 1994:38). La gravedad del problema ha sido causa de importantes modificaciones aprobadas en la última reforma laboral de este mismo año, sin embargo, dada su reciente implantación, es prematuro hacer valoraciones.

Desde el punto de vista del desarrollo humano de estos jóvenes, esta falta de opciones de trabajo, con la consiguiente carencia de ingresos, tiene repercusiones importantes en cuanto a las posibilidades 
de independencia de la familia de origen retrasando la formación de la propia familia y cambiando los roles de padres y abuelos que se ven obligados a seguir manteniendo a los hijos y, en muchas ocasiones, también a los nietos. Además, es fuente de problemas sociales como delincuencia, drogadicción, etc., por lo que el desempleo y subempleo juvenil puede calificarse de despilfarro de recursos humanos y amenaza para la cohesión económica y social (Comunidades Europeas, 1995).

En cuanto a la variable género, el colectivo de las mujeres es uno de los más castigados por el desempleo, lo que guarda estrecha relación con la situación social de la mujer española (Fernández Viguera, 1990:65-86). No hay que olvidar que, hasta hace relativamente poco, el nivel de estudios era muy bajo y la cualificación profesional prácticamente inaccesible para la mujer, las familias primaban la cualificación de los hijos varones como futuros «cabezas de familia», de la mujer se esperaba que se dedicara a «sus labores» familiares, abandonando el trabajo, si es que lo tenía, al contraer matrimonio. La consecuencia es que estas mujeres no han estado cotizando, su trabajo ha sido «invisible», por lo que han quedado dependientes de los ingresos del marido y desprotegidas cuando esta fuente de ingresos les fallaba: sin derecho a prestaciones sociales o éstas les resultan insuficientes, sin empleo cuando hay exceso de demanda, sin cualificación y, con frecuencia, con menores a su cargo.

Expertas/os de inserción laboral de las mujeres señalan, entre los obstáculos que estas encuentran: la alta precariedad en el empleo, su baja cualificación profesional, la absorción mayoritaria de empleos femeninos en la economía sumergida, los salarios inferiores a los hombres en el mismo puesto laboral, la elevada dificultad para acceder a puestos de responsabilidad, la acumulación del pluriempleo derivado de la doble jornada y la falta de autovaloración personal y profesional (Cáritas, 1995).

Las mujeres han venido estando sub-representadas en las cifras de beneficiarios de prestaciones sociales por desempleo, persistiendo en 1995 esta menor presencia femenina entre los beneficiarios de prestaciones, y, además, con menor nivel de protección ya que, en el caso de la prestaciones contributivas, la proporción es de una mujer por cada dos varones (CES, 1996:310-311). La menor protección social tiene relación con el mayor número de contratos a tiempo parcial, el subempleo y la discriminación de la mujer en caso de embarazo (Comisión de Política Social y Empleo del Congreso de los Diputados, 1994:8796), a pesar de los esfuerzos realizados por el Instituto de la Mujer. 
Especialmente difícil puede llegar a ser la situación de las mujeres solas cabezas de familia. En 1991, un 51\% de las mujeres separadas con hijos menores a su cargo, tenían ingresos anuales inferiores al millón de pesetas, así como dificultades de acceso a un empleo «normalizado» (Cáritas, 1991:62-66). Lo que explica que estas mujeres son uno de los grupos sobre-representados entre los que perciben las modalidades de renta mínima: «suponen entre 35 y un $45 \%$ del total, lo que resulta desproporcionado sobre su peso relativo en el conjunto de los hogares (alrededor del 10\%); parecen responder a dos grupos: mayores de 40-45 años, con más hijos y sin relación laboral anterior y otras mujeres más jóvenes (20-40) con menos hijos, con cierta experiencia laboral precaria y estudios primarios» (Aguilar, 1995:224).

Se acumulan, por una parte, todos los factores generales descritos que hacen muy difícil la inserción laboral de la mujer en general (mentalidad social tradicional del rol de ama de casa, la falta de cualificación profesional, el no tener experiencia laboral previa, etc.), a lo que se añaden otras dificultades ocasionadas por la asunción de rol de cabeza de familia, como la soledad ante la doble necesidad de conseguir ingresos para mantener el hogar y el cuidado de los menores a su cargo. En estas circunstancias, cuando la mujer se encuentra sin apoyos para el cuidado de los menores, se encuentra atrapada, ya que no puede capacitarse profesionalmente y/o no puede salir a trabajar por tener que encargarse de sus hijos. Por todo ello, la monoparentalidad es para la mujer un grave riesgo de inicio de un proceso de empobrecimiento y exclusión social.

Este mismo problema es extensivo a las mujeres que se ven obligadas a dedicarse al cuidado de las personas mayores dependientes de la familia, lo que resulta de gran actualidad ante el progresivo envejecimiento de la población, con las consiguientes mayores probabilidades de dependencia. Como señalan López de Lera e Izquierdo, «es necesario prevenir y evitar el aumento de la exclusión social en el ámbito de las mujeres ancianas, situación que en las grandes áreas metropolitanas es causa de un preocupante incremento de situaciones de abandono y pobreza entre este colectivo» (López de Lera e Izquierdo, 1995:25).

Esto es altamente injusto cuando tradicionalmente la mujer española ha tenido que asumir por imperativo sociocultural el cuidado de los miembros del hogar, lo que ha supuesto un importante ahorro al gasto público. El problema resulta de gran actualidad cuando la tendencia es fomentar la permanencia de estas personas en el hogar, lo que acaba queriendo decir que lo cuide la mujer de la casa, sin contar con su consentimiento (Rodríguez, 1994; Forster, 1994; Cerati, 1994). La mujer debe tener la oportunidad de elegir libremente si quiere ejer- 
cer ese rol de cuidadora, contando con apoyos públicos y, en el caso de que elija ese papel, el sistema de protección social debe compensarla suficientemente con vistas a su futuro, para prevenir posibles situaciones de pobreza o exclusión social.

\section{IV.EDUCANDO PARA LA PAZ, LUCHANDO CONTRA LA POBREZA Y LA EXCLUSIÓN SOCIAL.}

Como se ha venido diciendo, la pobreza y la exclusión social son formas de violencia estructural que pueden y deben ser evitadas y erradicadas. Las personas que carecen de oportunidades para su desarrollo personal y social sufren distintas formas de violencia directa que afectan directamente a su calidad de vida, violencia que se debe a la injusticia social perpetrada por el resto del grupo social que sí dispone de las oportunidades necesarias para su desarrollo humano.

A su vez, la pobreza y la exclusión social son factores de inestabilidad económica, política, social y cultural que pueden generar violencia física, siendo una amenaza real para las personas, tal como se está constatando en todo el mundo. No es de extrañar que las personas que se encuentran sin oportunidades o inseguros ante su futuro inmediato actúen violentamente cuando observan el contraste de las condiciones de vida de otras personas que, por tener poder de decisión, disponen de mucho más de lo necesario.

La respuesta ante este aumento de la violencia desde las políticas públicas y las élites de la sociedad no ha sido, hasta ahora, un cambio en las políticas macroeconómicas con el fin de mejorar la situación de las personas en situación de pobreza o exclusión social y prevenir el empeoramiento del problema. Por el contrario, se ha venido respondiendo a la violencia con más violencia desde las fuerzas de seguridad e incluso aumentando estas, en vez de atacar las causas que han generado esa ola de inseguridad personal a la que la gente sin acceso al poder no tiene más armas para responder que con la propia violencia. Las élites sociales reaccionan recurriendo a cuerpos privados de seguridad y exigiendo a los poderes públicos mayor represión y control de las fuerzas de seguridad pública.

Hay que recordar que, el verdadero desarrollo social, no es posible si no se favorece la plena integración social de TODAS las personas y que la exclusión y la discriminación no se producen solamente por problemas económicos sino también por diferencias de género, raciales, étnicas, físicas, psicológicas, sensoriales, etc.

Para que dicha integración social sea posible se precisan dos condiciones, por una parte, que el contexto social sea favorable a di- 
cha integración y, por otra, que las personas sean capaces de participar en la vida social, cultural, económica y política. Ambas están estrechamente relacionadas de tal manera que, si las personas no han tenido la oportunidad de desarrollar sus habilidades, no podrán aprovechar sus oportunidades de participar, por lo que se debe ofrecer las necesarias oportunidades, protegiendo a los más débiles y reconociendo el derecho a la diferencia para crear e innovar. Hay que capacitar a la población para poder vivir en común, respetar la dignidad de cada cual, primando la no violencia y la solidaridad.

Según Naciones Unidas, una «sociedad socialmente integrada» es: «Una sociedad capaz de dar cabida a aspiraciones individuales y de grupos diferentes y divergentes dentro de una estructura flexible de valores básicos compartidos e intereses comunes. Vista en el contexto del desarrollo humano sostenible, la integración social es sinónimo de más justicia, más igualdad, más bienestar material y más libertad democrática, lo que entraña igualdad de oportunidades y derechos para todos. En el seno de la sociedad se manifiesta como solidaridad, interdependencia, respeto a la diversidad cultural, tolerancia de los estilos de vida distintos de lo general y valor para sustituir sistemas que son inoperantes por otros más equitativos» (Naciones Unidas, 1994).

El desarrollo humano es interdependiente del crecimiento económico, pero este crecimiento debe tener las siguientes características: generar empleo y seguridad en los medios de ganarse el sustento; propiciar la libertad de las personas; distribuir equitativamente los beneficios; promover la cohesión social y la cooperación; salvaguardar el desarrollo humano futuro (PNUD, 1996:63). Este es un modelo de crecimiento muy diferente del modelo imperante, que al disociar los aspectos sociales y económicos del desarrollo esta generando pobreza y exclusión social, tal como se ha comentado, sin que ello sea inevitable (Riechman y Recio, 1997).

Concluyendo, la lucha contra la pobreza y la exclusión social implica la sensibilización de la sociedad sobre la existencia de las situaciones de violencia en que están inmersas las personas que se encuentran ante la pobreza o la exclusión social. Porque la mayoría de estas situaciones son ignoradas por resultar invisibles al resto de la población, unas veces por vergüenza de los que las sufren y otras veces por intereses políticos en invisibilizarlas. Si se desconocen, difícilmente se puede reconocer la injusticia social que comportan y menos ayudarles a reivindicar el reconocimiento de sus derechos.

Por lo tanto, es importante, en primer lugar, la investigación para conocer y hacer visibles estas situaciones que impiden a algunas personas su desarrollo humano. En segundo lugar, hay que actuar. La pobreza y la exclusión social pueden y deben evitarse, para lo que hay 
que plantear la lucha contra la pobreza o la exclusión como un tema de derechos humanos y de justicia social, lo que resituaría el debate sobre las políticas sociales y el papel del Estado. En todo ello, la Universidad, en su conjunto, no puede permanecer al margen. Y, en particular, el Trabajo Social debe replantear su rol ante los nuevos desafíos (Macarov, 1991:114-116), por ejemplo, con un mayor acercamiento entre lo económico y lo social (Midgley, 1996:13-25), para lo que se sugiere incidir en la formación de los futuros profesionales de ambos ámbitos desde la docencia universitaria (Martínez Román, 1996b).

Las políticas públicas no han sabido anticiparse ni responder a los nuevos problemas sociales, en particular, los Servicios Sociales deben replantear su rol (Martínez Román, 1997). Como señala la ONU, «lo que falta no son los recursos ni las soluciones económicas sino el impulso político para enfrentar directamente la pobreza» (PNUD, 1997:106), con tres elementos esenciales: potenciación política de los pobres, unión de actores sociales para el cambio y un Estado propicio y responsable.

Ante causas estructurales, no se puede recurrir a culpabilizar a las víctimas o a plantear actuaciones meramente asistenciales «in extremis» (Martínez Román y Guillén, 1996:334). El Estado no puede eludir su responsabilidad, aún cuando se reconozca la corresponsabilidad de la sociedad y se le reconozca su importante protagonismo. Los pobres deben organizarse para hacerse visibles y participar en la toma de decisiones que afectan directamente a sus vidas; el resto de la sociedad a través de las asociaciones de interés social, partidos políticos, los sindicatos, medios de comunicación, universidad, empresas, etc., necesitan unirse para potenciar un desarrollo humano. Pero todo este esfuerzo social precisa el apoyo de un Estado «activo y fuerte, que use su fuerza en favor de los pobres y no en su contra», que propicie espacios democráticos en los que la población «pueda articular sus demandas, actuar colectivamente y luchar por una distribución más equitativa del poder», asegurando que «las prácticas y los principios democráticos lleguen a todos los niveles y dimensiones de la sociedad» (PNUD, 1997:114-119).

\section{BIBLIOGRAFÍA}

AGUILAR, M. et al. La caña y el pez. Estudio sobre los salarios sociales en las Comunidades Autónomas. Fundación FOESSA, Madrid, 1995.

CÁRITAS ESPAÑOLA. «Pobreza y Desarrollo. Las condiciones de la nueva pobreza». Dossier, 1991, nº 25. 
CÁRITAS ESPAÑOLA. III Jornadas Estatales «Mujer y Trabajo». Programa de Mujer de los Servicios Sociales Generales. Cercedilla (Madrid) $17-$ 19 febrero, 1995.

CÁRITAS ESPAÑOLA. «Políticas contra la exclusión social». Documentación Social. Revista de Estudios Sociales y de Sociología Aplicada, 1997, $\mathrm{n}^{\circ} 106$, monográfico.

CASTEL, R. Les métamorphoses de la question sociale. Fayard, París, 1995. CENTRO DE ESTUDIOS DEL CAMBIO SOCIAL. Informe España 1996, una interpretación de su realidad social. Fundación Encuentro, Madrid, 1997.

CERATI, C. La mala hija. Anaya \& Mario Muchnik, Barcelona, (1994).

CES. España 1995. Economía, Trabajo y Sociedad. Memoría sobre la situación socioeconómica y laboral. Consejo Económico y Social, Madrid, 1996.

CES. España 1996. Economía, Trabajo y Sociedad. Memoría sobre la situación socioeconómica y laboral. Comité Económico y Social, Madrid, 1997.

COMISIÓN DE POLÍTICA SOCIAL Y EMPLEO DEL CONGRESO DE LOS DIPUTADOS, 4 de octubre de 1994. Diario de Sesiones del Congreso de los Diputados, V Legislatura, Comisiones, $\mathrm{n}^{\circ} 297$.

COMISIÓN SUDAMERICANA DE PAZ, SEGURIDAD Y DEMOCRACIA. «Propuestas emanadas de los «Foros Regionales de Actores sociales para promover una Agenda social en América Latina», Santiago de Chile, 5-6 diciembre, 1994.

COMISIÓN DE TRABAJO Y SEGURIDAD SOCIAL DEL SENADO, 19 de abril de 1994. Diario de Sesiones del Senado, V Legislatura, Comisiones, $n^{\circ} 95$.

COMUNIDADES EUROPEAS. Comité Económico y Social. «Dictámen del Comité Económico y Social sobre la «Exclusión Social». Bruselas. SOC/ 257. CES 1014/93, 1993.

COMUNIDADES EUROPEAS. Comité Económico y Social. «El paro Juvenil». Dictámen de Iniciativa de la Sección de Asuntos Sociales, Familia, Educación y Cultura. SOC/293, 1995.

ELVIRA, S. «Contrato de aprendizaje, así no». El País, 5 de febrero de 1994.

ENGELHARD, P. L'Homme Mondial. Les sociétés humaines peuvent-elles survivre. Arléa, Paris, 1996.

FERNÁNDEZ VIGUERA, B. «Pobreza femenina y estructura social», en VV.AA. Cuestiones en torno al Trabajo Social. Popular, Madrid, 1990, págs. 65-86.

FORSTER, M. Los hombres primero. Ediciones B., Barcelona, 1994.

GALTUNG, J. Sobre la paz. Fontamara, Barcelona, 1985. 
GALTUNG, J. Investigaciones teóricas. Sociedad y Cultura contemporáneas. Tecnos-Instituto de Cultura «Juan Gil-Albert», Madrid, 1995.

GALTUNG, J. Peace by peaceful means. Peace and Conflict, Development and Civilization. Sage-Prio, Londres, 1996.

LÓPEZ DE LERA, D. e Izquierdo Escribano, A. «Transformaciones demográficas y nuevas formas de convivencia en la población española». Sociedad y Utopía. Revista de Ciencias Sociales, 1995, nº 6, pág. 25.

MACAROV, D. Certain Change. Social Work practice in the future. NASW Press, Silver Spring-MD, 1991.

MARTÍNEZ ROMÁN, $\mathrm{M}^{\mathrm{a}}$ A. Exclusión y Política social: respuestas públicas a las nuevas necesidades sociales en España y Reino Unido. (Tesis Doctoral becada por el Instituto de Estudio Juan Gil-Albert). Universidad de Alicante, Alicante, 1996a.

MARTÍNEZ ROMÁN, Mª A. «Algunos problemas de las políticas públicas de empleo dirigidas a los colectivos con mayores dificultades de inserción». IV Encuentro Internacional sobre Servicios Sociales «Exclusión e Intervención social», Valencia 29-31 de octubre, 1996b.

MARTÍNEZ ROMÁN, $M^{a}$ A. «Los Servicios Sociales públicos y la exclusión social», La Cristalera. Revista de Asuntos Sociales, Dirección General de Bienestar Social-Murcia, 1997, nº 7.

MARTÍNEZ ROMÁN, Ma A. y GUILLÉN, E. «Estado de Bienestar y Servicios Sociales: problemas, reacciones y medidas necesarias» en Casilda, $\mathbf{R}$. y Tortosa, J.M. (Editores) Pros y contras del Estado de Bienestar. Tecnos, Madrid, 1996, págs. 313-336.

MERVA, M. y FOWLES, R. Effects of disminished economic opportunities on social stress: heart attacks, strokes and crime. Economic Policy Institute, Washington, DC, 1992.

MIDGLEY, J. «Involving Social Work in economic development». International Social Work, 1996, vol. 39, n 1, págs. 13-25.

NACIONES UNIDAS. «Hacía una Sociedad para Todos». Documento de Antecedentes $n^{\circ} 3$ de la Cumbre Mundial sobre Desarrollo Social, Copenhague, 6-12 marzo, 1995.

NACIONES UNIDAS. «Conferencia Internacional sobre la Población y el Desarrollo», El Cairo, 5-13 septiembre 1994. A/CONF:171/13.

OIT. El empleo en el mundo 1996-97: las políticas nacionales a la hora de la mundialización. Organización Internacional del Trabajo, Ginebra, 1997.

PÉREZ, E. «Trabajadores de segunda categoría». Cáritas, 1996, n 58.

PNUD. Informe sobre Desarrollo Humano 1994. Fondo de Cultura Económica, México, 1995.

PNUD. Informe sobre Desarrollo Humano 1996. Mundi-Prensa, Madrid, 1996. 
PNUD. Informe sobre Desarrollo Humano 1997. Mundi- Prensa, Madrid, 1997.

QUEVEDO AGUADO, M.P. «La propuesta de la Renta Mínima y el Salario Ciudadano desde un modelo constructivista de la personalidad en el medio psicosocial». Documentación Social, 1990, n 78, enero-marzo.

RECIO, A. Trabajo, personas, mercados. Manual de Economía Laboral. Icaria-FUHEM, Barcelona, 1997.

RIECHMAN, J. y RECIO, A. Quien parte y reparte... El debate sobre la reducción del tiempo del trabajo. Icaria, Barcelona, 1997.

RIFKIN, J. El fin del trabajo. Nuevas tecnologias contra puestos de trabajo: el nacimiento de una nueva era. Paidós, Barcelona, 1996.

RODGERS, G. «What is special about a «social exclusion» approach?», en Rodgers, G., Gore, C. y Figuereido, J.B. Social Exclusion: Rhetoric, Realty, Responses. ILO, Ginebra, 1995.

RODRÍGUEZ, J.A. Envejecimiento y familia. CIS, Madrid, 1994.

TORTOSA, J.M. La pobreza capitalista. Tecnos, Madrid, 1993. 